\title{
ON A THEOREM OF BREIMAN AND A CLASS OF RANDOM DIFFERENCE EQUATIONS
}

\author{
DENIS DENISOV, ${ }^{*}$ EURANDOM \\ BERT ZWART, ${ }^{* *}$ Georgia Institute of Technology
}

\begin{abstract}
We consider the tail behavior of the product of two independent nonnegative random variables $X$ and $Y$. Breiman (1965) has considered this problem, assuming that $X$ is regularly varying with index $\alpha$ and that $\mathrm{E}\left\{Y^{\alpha+\varepsilon}\right\}<\infty$ for some $\varepsilon>0$. We investigate when the condition on $Y$ can be weakened and apply our findings to analyze a class of random difference equations.
\end{abstract}

Keywords: Regular variation; subexponential distribution; random difference equation

2000 Mathematics Subject Classification: Primary 60H25; 60J30; 60F10

\section{Introduction}

Suppose that $X$ and $Y$ are two independent nonnegative random variables such that $\mathrm{P}\{X>x\}$ is regularly varying with index $-\alpha, \alpha \geq 0$ (i.e. $\mathrm{P}\{X>y x\} / \mathrm{P}\{X>x\} \rightarrow y^{-\alpha}$ as $x \rightarrow \infty$ ), and that $\mathrm{E}\left\{Y^{\alpha+\varepsilon}\right\}<\infty$ for some $\varepsilon>0$. Then

$$
\mathrm{P}\{X Y>x\} \sim \mathrm{E}\left\{Y^{\alpha}\right\} \mathrm{P}\{X>x\} \quad \text { as } x \rightarrow \infty,
$$

with $f(x) \sim g(x)$ denoting $f(x)=g(x)(1+\mathrm{o}(1))$. This result was first stated by Breiman [2] for $\alpha \in[0,1]$, and is known as Breiman's theorem; a more recent study containing a proof for all $\alpha$ can be found in [5].

We are interested in extensions of (1.1), in particular, in relaxing the condition $\mathrm{E}\left\{Y^{\alpha+\varepsilon}\right\}<\infty$. Apart from its intrinsic interest, our motivation for this comes from the well-known random affine equation

$$
R \stackrel{\mathrm{D}}{=} M R+Q
$$

where ' $\stackrel{\text { D, }}{=}$ denotes equality in distribution and $M, Q$, and $R$ are random variables independent of each other. This equation appears in many different applications, most notably in actuarial and financial mathematics. If $\mathrm{P}(|M|>1)>0$ then $R$ typically has a power tail and this case is fairly well understood; classical papers are [10] and [14]. Unfortunately, not many results are available when $Q$ is light tailed and $|M| \leq 1$. Some partial results can be found in [11] and [19]. A relatively clean case seems to be when $\mathrm{e}^{Q}$ is regularly varying with index $-\alpha$ and independent of $M$. After taking exponents in (1.2), we may wonder whether the application of Breiman's theorem is justified, i.e. whether $\mathrm{P}\{R>x\} \sim \mathrm{E}\left\{\mathrm{e}^{\alpha M R}\right\} \mathrm{P}\{Q>x\}$. If $|M|<1-\delta$

Received 21 November 2005; revision received 1 November 2007.

* Current address: School of Mathematical and Computer Sciences, Heriot-Watt University, Edinburgh EH14 4AS, UK. Email address: denisov@ma.hw.ac.uk

** Postal address: H. Milton Stewart School of Industrial and Systems Engineering, Georgia Institute of Technology, 765 Ferst Drive, Atlanta, GA 30332-0205, USA. 
almost surely (a.s.) for some $\delta>0$ then we can show that $\mathrm{E}\{\exp ((\alpha+\varepsilon) M R)\}<\infty$ for some $\varepsilon>0$, so that Breiman's theorem (1.1) can indeed be applied. However, assuming the existence of such a $\delta>0$ is not very natural; we are interested in the extent to which the tail equivalence between $R$ and $Q$ remains true without invoking such an assumption. To obtain an answer to this question, the conditions under which Breiman's theorem remains true need to be relaxed along the lines mentioned above.

We now proceed with an informal presentation of our results. If $X$ is regularly varying with index $-\alpha$, the most general conditions on $Y$ under which (1.1) holds would be

$$
\mathrm{E}\left\{Y^{\alpha}\right\}<\infty \quad \text { and } \quad \mathrm{P}\{Y>x\}=\mathrm{o}(\mathrm{P}\{X>x\}) .
$$

We show that this set of conditions on $Y$ is in general not enough for (1.1) to hold. To obtain sufficient conditions, we mainly focus on invoking additional assumptions on the slowly varying function $L$ in the representation $\mathrm{P}\{X>x\}=L(x) x^{-\alpha}$. In particular, we consider the following three different cases.

1. If $L(x)$ is bounded away from 0 (in a sense we make precise later on) then (1.3) implies (1.1) without any further assumptions.

2. If $L(x)$ is eventually decreasing to 0 then, for large enough $x, L\left(\mathrm{e}^{x}\right)=\mathrm{P}\{U>x\}$ for some long-tailed random variable $U$. It turns out that the additional condition $U \in \delta^{*}$ is crucial for (1.1) to hold if $U$ has a finite mean. A similar type of assumption has to be made if $L(x)$ oscillates at $\infty$; we review $\delta^{*}$ and other classes of distributions in Section 2.

3. If the condition $\mathrm{E}\{U\}<\infty$ in the previous case does not hold (which is the case when $\mathrm{E}\left\{X^{\alpha}\right\}=\infty$ and $\lim _{x \rightarrow \infty} x^{\alpha} \mathrm{P}\{X>x\}=0$ ) then we also need to invoke an additional condition to ensure the validity of (1.1).

These three cases are respectively covered by Propositions 2.1-2.3 in Section 2. The necessity of several of the additional regularity conditions is illustrated by a number of counterexamples in Section 3.

The results of the present paper are related to several existing results in the literature. Several researchers independently showed that (1.1) always holds if $\mathrm{P}\{X>x\} \sim c x^{-\alpha}$ and $\mathrm{E}\left\{Y^{\alpha}\right\}<\infty$; see, e.g. [12, Lemma 2.1] and [19, Lemma 5.1]. Our Proposition 2.1 is an extension of these results. Embrechts and Goldie [7] showed that $X Y$ is regularly varying with index $-\alpha$ if both $X$ and $Y$ are regularly varying with index $-\alpha$, without providing explicit asymptotics. Cline [3] contains a property of the class $\delta(\gamma)$ which is strongly related to the second case discussed above; we come back to this in Section 2. In addition, Cline [3] investigated the asymptotic behavior of $\mathrm{P}\{X Y>x\}$ in various cases where $\mathrm{P}\{X Y>x\} / \mathrm{P}\{X>x\}$ $\rightarrow \infty$.

This paper is organized as follows. In Section 2 we give a number of sufficient conditions on $L$ in order for (1.1) to hold under (1.3). Counterexamples are provided in Section 3. In Section 4 we apply our results to obtain the tail behavior of $R$ in the random difference equation mentioned above. Some concluding remarks are given in Section 5.

\section{Extensions of Breiman's theorem}

In this section we investigate which assumptions, in addition to (1.3), are needed to guarantee (1.1). To make the paper self contained, we first give a quick review of the classes of heavy-tailed distribution functions which are considered in this section. 
A function $L$ is slowly varying if $L(a x) / L(x) \rightarrow 1$ for any $a>0$ as $x \rightarrow \infty$. A random variable $X$ has a regularly varying distribution tail if there exists a slowly varying function $L$ and a constant $\alpha \geq 0$ such that $\mathrm{P}\{X>x\}=L(x) x^{-\alpha}$. We often summarize this as $X$ being regularly varying with index $-\alpha$.

A random variable $X$ is long tailed (we write $X \in \mathcal{L}$ ) if $\mathrm{P}\{X>x\} \sim \mathrm{P}\{X>x+y\}$ as $x$ tends to $\infty$ for any $y$. Note that the function $\mathrm{P}\{X>\log x\}$ is slowly varying if $X$ is long tailed.

An additional class of densities is defined as follows. A nonnegative function $f$ is in the class $\wp_{d}$ (and in this case we call $f$ a subexponential density) if it satisfies the property

$$
\lim _{x \rightarrow \infty} \int_{0}^{x} \frac{f(x-y)}{f(x)} f(y) \mathrm{d} y=2 \int_{0}^{\infty} f(u) \mathrm{d} u<\infty .
$$

If $f(x)=\mathrm{P}\{U>x\}$ for some random variable $U$, we say that $U \in 8^{*}$. Both classes $\delta_{d}$ and $\varsigma^{*}$ have been introduced by Klüppelberg [15], [16].

Finally, recall that a nonnegative random variable $T$ is in the class $\delta(\gamma), \gamma \geq 0$, if, as $x \rightarrow \infty$,

$$
\frac{\mathrm{P}\{T>x+y\}}{\mathrm{P}\{T>x\}} \rightarrow \mathrm{e}^{-\gamma y} \text { and } \frac{\mathrm{P}\left\{T+T^{\prime}>x\right\}}{\mathrm{P}\{T>x\}} \rightarrow 2 \mathrm{E}\left\{\mathrm{e}^{\gamma T}\right\}<\infty,
$$

with $T^{\prime}$ an independent and identically distributed (i.i.d.) copy of $T$. If $\gamma=0$, we recover the class $\delta$ of subexponential distributions. The class $\delta^{*}$ is a subclass of $\delta$. In [16] it was shown that, for $\gamma>0, T \in \delta(\gamma)$ if and only if $\mathrm{e}^{\gamma x} \mathrm{P}\{T>x\}$ is in $\varsigma_{d}$. A recent interesting paper on these classes of distributions is [9]. Finally, a function $f$ is of dominated variation if

$$
0<\liminf _{x \rightarrow \infty} \frac{f(2 x)}{f(x)}<\limsup _{x \rightarrow \infty} \frac{f(2 x)}{f(x)}<\infty .
$$

For more background on heavy tails, we refer the reader to [8].

As a preliminary we give a representation of slowly varying functions in terms of long-tailed distribution functions. Throughout the rest of the paper, we use various properties of regularly varying functions which all appear in the monograph [1].

Lemma 2.1. Let $L$ be slowly varying. Then $L$ admits precisely one of the following four representations:

(i) $L(x)=c(x)$,

(ii) $L(x)=c(x) / \mathrm{P}\{V>\log x\}$,

(iii) $L(x)=c(x) \mathrm{P}\{U>\log x\}$,

(iv) $L(x)=c(x) \mathrm{P}\{U>\log x\} / \mathrm{P}\{V>\log x\}$.

In all representations, $c(x)$ is a function converging to a constant $c \in(0, \infty)$. Here $U$ and $V$ are two independent long-tailed random variables with hazard rates converging to 0.

Throughout this paper, the random variables $U$ and $V$ appearing in Lemma 2.1 will be referred to as auxiliary random variables. 
Proof of Lemma 2.1. By the representation theorem for slowly varying functions we can write, for some function $c(x) \rightarrow c \in(0, \infty)$ and $h(x) \rightarrow 0$,

$$
L(x)=c(x) \exp \left(\int_{1}^{x} \frac{h(u)}{u} \mathrm{~d} u\right) .
$$

Write $h(u)=h^{+}(u)-h^{-}(u)$, with $h^{+}(u)$ denoting the positive part of $h(u)$ and $h^{-}(u)$ denoting the negative part of $h(u)$. Both $h^{+}(u)$ and $h^{-}(u)$ converge to 0 . A first issue to consider is whether $\int_{1}^{x} h^{i}(u) / u \mathrm{~d} u$ converges, where $i=+,-$. If this would be the case, then this can be incorporated in the function $c(x)$, so without loss of generality, we can assume that either $h^{i}(u)=0$ or the corresponding integral diverges. This leads to the four cases of Lemma 2.1.

Now suppose that $\int_{1}^{x} h^{+}(u) / u \mathrm{~d} u$ diverges. Then there exists a long-tailed random variable $V$ such that

$$
\exp \left(-\int_{1}^{x} \frac{h^{+}(u)}{u} \mathrm{~d} u\right)=\exp \left(-\int_{0}^{\log x} h^{+}\left(\mathrm{e}^{v}\right) \mathrm{d} v\right)=\mathrm{P}\{V>\log x\}
$$

A similar argument can be made for $h^{-}$.

Proposition 2.1, below, states our first sufficient condition for (1.1), and its assumption on $L$ covers cases (i) and (ii) of Lemma 2.1.

Proposition 2.1. Assume that, in addition to (1.3), $\lim _{\sup } \rightarrow \infty \sup _{1 \leq y \leq x} L(y) / L(x)<\infty$. Then (1.1) holds.

Proof. The assumption on $L$ implies that it is bounded away from 0 , which implies that $\alpha>0$. By replacing $X$ and $Y$ with $X^{\alpha}$ and $Y^{\alpha}$, respectively, if $\alpha \neq 1$, we can assume that $\alpha=1$. We can also assume, without loss of generality, that $\mathrm{P}\{Y=0\}=0$. Note that the asymptotic lower bound

$$
\liminf _{x \rightarrow \infty} \frac{\mathrm{P}\{X Y>x\}}{\mathrm{P}\{X>x\}} \geq \mathrm{E}\{Y\}
$$

always holds in view of Fatou's lemma. To obtain an upper bound, write

$$
\mathrm{P}\{X Y>x\}=\sum_{i=1}^{4} \mathrm{P}\left\{X Y>x ; Y \in A_{i}\right\}
$$

with $A_{1}=[0, \varepsilon), A_{2}=[\varepsilon, M), A_{3}=[M, g(x) x)$, and $A_{4}=[g(x) x, \infty)$. Here, $g(x) \downarrow 0$ is chosen such that $\mathrm{P}\{Y>g(x) x\}=\mathrm{o}(\mathrm{P}\{X>x\})$ and $g(x) x \rightarrow \infty$. Number the four terms as $I_{1}, \ldots, I_{4}$. Then

$$
I_{1} \leq \mathrm{P}\left\{X>\frac{x}{\varepsilon}\right\} \sim \varepsilon \mathrm{P}\{X>x\}
$$

Furthermore, by the uniform convergence theorem for slowly varying functions we obtain

$$
I_{2} \sim \mathrm{E}\{Y ; \varepsilon<Y<M\} \mathrm{P}\{X>x\}
$$


The fourth term can be upper bounded as follows:

$$
I_{4} \leq \mathrm{P}\{Y>g(x) x\}=\mathrm{o}(\mathrm{P}\{X>x\}) .
$$

Thus, it remains to consider

$$
I_{3}=\mathrm{P}\{X>x\} \int_{M}^{g(x) x} \frac{L(x / y)}{L(x)} y \mathrm{dP}\{Y \leq y\} .
$$

We have, for $C(x)=\sup _{y \in[1, x]} L(y) / L(x)=\sup _{y \in[1, x]} L(x / y) / L(x)$,

$$
I_{3} \leq \mathrm{P}\{X>x\} \sup _{y \in[1, x]} \frac{L(x / y)}{L(x)} \mathrm{E}\{Y ; Y>M\} \sim C(x) \mathrm{P}\{X>x\} \mathrm{E}\{Y ; Y>M\} .
$$

Putting everything together, we obtain

$$
\limsup _{x \rightarrow \infty} \frac{\mathrm{P}\{X Y>x\}}{\mathrm{P}\{X>x\}} \leq \varepsilon+\mathrm{E}\{Y ; \varepsilon<Y<M\}+C(\infty) \mathrm{E}\{Y ; Y>M\},
$$

with $C(\infty):=\lim _{\sup _{x \rightarrow \infty}} C(x)$. The result now follows by noting that $C(\infty)<\infty$ and by letting $\varepsilon \downarrow 0$ and $M \rightarrow \infty$.

Note that the assumption on $L$ was used only in the estimate of term $I_{3}$; the estimates of all other terms hold under (1.3). We now investigate what happens in cases (iii) and (iv) of Lemma 2.1. It turns out that the situation is more complicated in these cases. In particular, we need to rely on properties of the classes $\S_{d}$ and $\delta^{*}$ to estimate $I_{3}$.

Proposition 2.2. Assume, in addition to (1.3), that $\alpha>0$ and that $L$ admits representation (iii) or (iv) of Lemma 2.1. If either $L\left(\mathrm{e}^{x}\right) \in \varsigma_{d}$ or the auxiliary random variable $U \in \delta^{*}$ and $\mathrm{P}\{Y>y\}=\mathrm{o}\left(y^{-\alpha} \mathrm{P}\{U>\log y\}\right)$ then (1.1) holds.

Note that the condition $\mathrm{P}\{Y>y\}=\mathrm{o}\left(y^{-\alpha} \mathrm{P}\{U>\log y\}\right)$ holds in case (iii) of Lemma 2.1.

Proof of Proposition 2.2. If $L\left(\mathrm{e}^{x}\right) \in \delta_{d}$ then, according to Theorem 2.1 of [16], $\log X \in$ $\delta(\gamma)$ and the result then follows from [3]; see the discussion below Theorem 1 of [3].

For the other case, we proceed similarly as in the proof of Proposition 2.1. We again assume, without loss of generality, that $\alpha=1$. After this transformation, the auxiliary random variables $U$ and $V$ in the representation of $L$ are replaced by $\alpha U$ and $\alpha V$, respectively, which causes no additional complications, for example, $\alpha U \in \delta^{*}$ if and only if $U \in \delta^{*}$. It remains to estimate $I_{3}$. Define $c^{*}(x)=\sup _{y \in[M, g(x) x]} c(x / y) / c(x)$. Note that $c^{*}(x)$ is bounded since $c(x)$ converges in $(0, \infty)$. Observe that

$$
\begin{aligned}
I_{3} & =\mathrm{P}\{X>x\} \int_{M}^{g(x) x} \frac{L(x / y)}{L(x)} y \mathrm{dP}\{Y \leq y\} \\
& \leq \mathrm{P}\{X>x\} c^{*}(x) \int_{M}^{g(x) x} \frac{\mathrm{P}\{U>\log x-\log y\}}{\mathrm{P}\{U>\log x\}} y \mathrm{dP}\{Y \leq y\} .
\end{aligned}
$$

It suffices to show that

$$
\lim _{M \rightarrow \infty} \limsup _{x \rightarrow \infty} \int_{M}^{g(x) x} \frac{\mathrm{P}\{U>\log x-\log y\}}{\mathrm{P}\{U>\log x\}} y \mathrm{dP}\{Y \leq y\}=0 .
$$


Let $s(x)=\mathrm{P}\{Y>x\} / x^{-1} \mathrm{P}\{U>\log x\} \rightarrow 0$. Integrating by parts, we obtain

$$
\begin{array}{rl}
\int_{M}^{g(x) x} & \mathrm{P}\{U>\log x-\log y\} y \mathrm{dP}\{Y \leq y\} \\
= & -\mathrm{P}\{Y>g(x) x\} \mathrm{P}\{U>-\log g(x)\} x g(x)+\mathrm{P}\{Y>M\} M \mathrm{P}\{U>\log x-\log M\} \\
& +\int_{M}^{x g(x)} \mathrm{P}\{U>\log x-\log y\} \mathrm{P}\{Y>y\} \mathrm{d} y \\
& +\int_{M}^{x g(x)} \mathrm{P}\{Y>y\} y \mathrm{~d}_{y} \mathrm{P}\{U>\log x-\log y\} .
\end{array}
$$

We continue by bounding all terms on the right-hand side of this expression. The first term is nonpositive and can therefore be discarded. To bound the second term, note that, since $U \in 8^{*} \subset \mathcal{L}$

$$
\lim _{x \rightarrow \infty} \frac{\mathrm{P}\{Y>M\} M \mathrm{P}\{U>\log x-\log M\}}{\mathrm{P}\{U>\log x\}}=\mathrm{P}\{Y>M\} M .
$$

For the third term, we have

$$
\begin{aligned}
& \limsup _{x \rightarrow \infty} \frac{\int_{M}^{x g(x)} \mathrm{P}\{U>\log x-\log y\} \mathrm{P}\{Y>y\} \mathrm{d} y}{\mathrm{P}\{U>\log x\}} \\
& \quad \leq \limsup _{x \rightarrow \infty} \sup _{y \geq M} s(y) \frac{\int_{M}^{x} \mathrm{P}\{U>\log x-\log y\} \mathrm{P}\{U>\log y\} \mathrm{d} \log y}{\mathrm{P}\{U>\log x\}} \\
& \quad \leq \sup _{y \geq M} s(y) 2 \mathrm{E}\{U\},
\end{aligned}
$$

since $U \in \varsigma^{*}$. Finally we have, for the fourth term,

$$
\begin{aligned}
& \limsup _{x \rightarrow \infty} \frac{\int_{M}^{x g(x)} \mathrm{P}\{Y>y\} y \mathrm{~d}_{y} \mathrm{P}\{U>\log x-\log y\}}{\mathrm{P}\{U>\log x\}} \\
& \quad \leq \limsup _{x \rightarrow \infty} \sup _{y \geq M} s(y) \frac{\int_{M}^{x} \mathrm{P}\{U>\log y\} \mathrm{d}_{y} \mathrm{P}\{U>\log x-\log y\}}{\mathrm{P}\{U>\log x\}} \\
& \quad \leq 2 \sup _{y \geq M} s(y),
\end{aligned}
$$

since $U \in 8^{*}$, and therefore $U$ is subexponential. Putting everything together, we see that

$$
\begin{aligned}
& \limsup _{x \rightarrow \infty} \int_{M}^{g(x) x} \frac{\mathrm{P}\{U>\log x-\log y\}}{\mathrm{P}\{U>\log x\}} y \mathrm{dP}\{Y \leq y\} \\
& \leq \mathrm{P}\{Y>M\} M+\sup _{y \geq M} s(y) 2 \mathrm{E}\{U\}+2 \sup _{y \geq M} s(y) .
\end{aligned}
$$

This converges to 0 if $M$ tends to $\infty$, since our assumption on $\mathrm{P}\{Y>y\}$ implies that $s(y) \rightarrow 0$. We conclude that (2.1) holds.

Finally note that the two assumptions $U \in 8^{*}$ and $L\left(\mathrm{e}^{x}\right) \in \delta_{d}$ are equivalent if case (iii) of Lemma 2.1 applies. However, in general, the two assumptions are not implied by one another. 
We continue by investigating a third case, which occurs when the auxiliary random variable $U$ has infinite mean, in which case $U$ cannot be in $8^{*}$. Set

$$
m(x)=\int_{0}^{x} t^{\alpha} \mathrm{dP}\{X \leq t\} .
$$

It is clear that since $\mathrm{E}\left\{X^{\alpha}\right\}=\infty$ if $\mathrm{E}\{U\}=\infty$, we have $m(x) \rightarrow \infty$.

Proposition 2.3. Assume, in addition to (1.3), that $\alpha>0$ and that

$$
\frac{\mathrm{P}\{Y>x\}}{\mathrm{P}\{X>x\}} m(x) \rightarrow 0 .
$$

Let $L$ be such that

$$
\limsup _{x \rightarrow \infty} \sup _{\sqrt{x} \leq y \leq x} \frac{L(y)}{L(x)}<\infty
$$

Then (1.1) holds.

A sufficient condition for (2.3) to hold is that $L\left(\mathrm{e}^{x}\right)$ is of dominated variation. The quantity $\sqrt{x}$ in (2.3) may be replaced by $x^{\beta}, \beta<1$.

Proof of Proposition 2.3. By considering $X^{\alpha}$ and $Y^{\alpha}$ when $\alpha \neq 1$, it suffices to prove the result for $\alpha=1$. We proceed similarly as in the proof of Proposition 2.2. It remains to estimate $I_{3}$ :

$$
I_{3}=\mathrm{P}\{X>x\} \int_{M}^{g(x) x} \frac{L(x / y)}{L(x)} y \mathrm{dP}\{Y \leq y\} .
$$

Since $\mathrm{P}\{Y>x\}=\mathrm{o}(\mathrm{P}\{X>x\})$, there exists a function $g_{1}(x) \downarrow 0$ such that $\mathrm{P}\left\{Y>g_{1}(x) x\right\}=$ $\mathrm{o}(\mathrm{P}\{X>x\})$. Set $g(x)=\max \left(2 / \sqrt{x}, g_{1}(x)\right) \geq g_{1}(x)$. Then $g(x) \downarrow 0$ and

$$
\mathrm{P}\{Y>g(x) x\} \leq \mathrm{P}\left\{Y>g_{1}(x) x\right\}=\mathrm{o}(\mathrm{P}\{X>x\}) .
$$

Therefore, we can assume that the function $g$ in $I_{3}$ has the properties $g(x) \downarrow 0$ and $g(x)>1 / \sqrt{x}$.

In what follows, we split the integral in $I_{3}$ into two integrals (according to the intervals $[M, \sqrt{x}]$ and $[\sqrt{x}, g(x) x])$ and estimate both of them separately.

For the first integral, we have

$$
\int_{M}^{\sqrt{x}} \frac{L(x / y)}{L(x)} y \mathrm{dP}\{Y \leq y\} \leq \sup _{\sqrt{x} \leq y \leq x} \frac{L(y)}{L(x)} \int_{M}^{\infty} y \mathrm{dP}\{Y \leq y\} .
$$

As $M$ goes to infinity, the latter goes to 0 , since $\mathrm{E}\{Y\}<\infty$ and condition (2.3) holds. We integrate the second integral by parts to obtain

$$
\begin{aligned}
\int_{\sqrt{x}}^{g(x) x} L\left(\frac{x}{y}\right) y \mathrm{dP}\{Y \leq y\}= & -\mathrm{P}\{Y>g(x) x\} L\left(\frac{1}{g(x)}\right) x g(x) \\
& +\mathrm{P}\{Y>\sqrt{x}\} \sqrt{x} L(\sqrt{x})+\int_{\sqrt{x}}^{x g(x)} \mathrm{P}\{Y>y\} \mathrm{d}_{y}\left(y L\left(\frac{x}{y}\right)\right) .
\end{aligned}
$$

The first term is nonpositive. For the second term, we have, owing to $\mathrm{E}\{Y\}<\infty$ and (2.3),

$$
\lim _{x \rightarrow \infty} \frac{P\{Y>\sqrt{x}\} \sqrt{x} L(\sqrt{x})}{L(x)}=0 .
$$


By (2.2), the third term satisfies

$$
\begin{aligned}
\frac{\int_{\sqrt{x}}^{x g(x)} \mathrm{P}\{Y>y\} \mathrm{d}(y L(x / y))}{L(x)} & =\mathrm{o}(1) \frac{\int_{\sqrt{x}}^{x g(x)} x(\mathrm{P}\{X>y\} / m(y)) \mathrm{dP}\{X \leq x / y\}}{L(x)} \\
& =\frac{\mathrm{o}(1)}{m(\sqrt{x})} \frac{\int_{\sqrt{x}}^{x g(x)} L(y) x y^{-1} \mathrm{dP}\{X \leq x / y\}}{L(x)} \\
& =\mathrm{o}(1) \frac{1}{m(\sqrt{x})} \sup _{\sqrt{x} \leq y \leq x} \frac{L(y)}{L(x)} \int_{1 / g(x)}^{\sqrt{x}} t \mathrm{dP}\{X \leq t\} \\
& =\mathrm{o}(1) \frac{m(\sqrt{x})-m(1 / g(x))}{m(\sqrt{x})} \sup _{\sqrt{x} \leq y \leq x} \frac{L(y)}{L(x)} \\
& \rightarrow 0,
\end{aligned}
$$

owing to condition (2.3).

Note that it is not sufficient to just assume that $\mathrm{P}\{Y>x\} / \mathrm{P}\{X>x\} \rightarrow 0$. This is illustrated with a counterexample in Section 3.

For completeness, we finally state a well-known result for $\alpha=0$.

Proposition 2.4. (Embrechts and Goldie [7].) Suppose, in addition to (1.3), that $\alpha=0$ and that, in particular, $\log X \in$ \&. Then (1.1) holds.

The fact that it is difficult to remove the assumption $\log X \in \delta$ is illustrated in Section 3.2.

\section{Counterexamples}

In Section 2 we saw that Breiman's theorem can be extended in a number of cases, but that the minimal conditions, (1.3), were not achieved. The goal of the present section is to illustrate that it is hard or even impossible to weaken the assumptions made in Propositions 2.2-2.4. In the next three subsections we give a counterexample related to each of these three propositions.

\subsection{A counterexample related to Proposition 2.2}

In this subsection we construct independent nonnegative random variables $X$ and $Y$ such that $X$ is regularly varying and $Y$ satisfies (1.3), but for which (1.1) fails.

Since $\alpha>0$, we can take $\alpha=1$ without loss of generality. Let $a(x)$ be a distribution tail which is long tailed, but not in $8^{*}$. Assume that $\int_{0}^{\infty} a(x) \mathrm{d} x<\infty$ and that

$$
\limsup _{x \rightarrow \infty} \int_{0}^{x} \frac{a(y) a(x-y)}{a(x)} \mathrm{d} y=\infty .
$$

(All known examples of distributions in $\mathcal{L} \backslash \&$ satisfy this property.) Since $a(x)$ is long tailed, but not in $8^{*}$, there exists a function $h(x)$ such that $h(x) \rightarrow \infty, a(x-h(x)) \sim a(x)$, and

$$
\limsup _{x \rightarrow \infty} \int_{h(x)}^{x-h(x)} \frac{a(y) a(x-y)}{a(x)} \mathrm{d} y=\infty .
$$


Now set $L(x)=a(\log x)$. From the previous considerations, it follows that there exists a function $d(x)$ such that we have $d(x) \rightarrow \infty, d(x)=\mathrm{o}(\sqrt{x}), L(x / d(x)) \sim L(x), \int_{1}^{\infty}(L(x) / x) \mathrm{d} x$ $<\infty$, and

$$
\limsup _{x \rightarrow \infty} \int_{d(x)}^{x / d(x)} \frac{L(y) L(x / y)}{y L(x)} \mathrm{d} y=: \limsup _{x \rightarrow \infty} r(x)=\infty .
$$

Let $x_{n}, n \geq 1$, be a sequence such that $x_{n} \rightarrow \infty$ and $r\left(x_{n}\right) \rightarrow \infty$.

We are now ready to define $X$ and $Y$. Let $X$ be a random variable with tail $L(x) / x$. Observe that $X$ has finite mean. Set, for $x \geq 0, s(x)=\sqrt{r\left(x_{n}\right)}$ when $x \in\left[d\left(x_{n}\right), d\left(x_{n+1}\right)\right)$. Observe that $s(x) \rightarrow \infty$. Let $g(x)=\mathrm{P}\{X>x\} / x s(x)=L(x) / x^{2} s(x)$ be the density of $Y$. Since $s(x) \rightarrow \infty$, it can be shown, by Karamata's theorem, that $\mathrm{P}\{Y>x\}=\mathrm{o}(\mathrm{P}\{X>x\})$.

Then

$$
\begin{aligned}
\frac{\mathrm{P}\{X Y>x ; d(x)<Y<x / d(x)\}}{\mathrm{P}\{X>x\}} & =\int_{d(x)}^{x / d(x)} \frac{L(y) L(x / y)}{y s(y) L(x)} \mathrm{d} y \\
& \geq \int_{d(x)}^{x / d(x)} \frac{L(y) L(x / y)}{y s(d(x)) L(x)} \mathrm{d} y \\
& =\frac{r(x)}{s(d(x))} .
\end{aligned}
$$

This is at least $\sqrt{r\left(x_{n}\right)}$ at the points $x_{n}, n \geq 1$. We conclude that

$$
\limsup _{x \rightarrow \infty} \frac{\mathrm{P}\{X Y>x ; Y>d(x)\}}{\mathrm{P}\{X>x\}}=\infty,
$$

implying that (1.1) does not hold. This illustrates that the condition $U \in 8^{*}$ in Proposition 2.2 cannot be weakened in general. We would like to remark that in the above construction we can additionally assume that both $a(x)$ and $\int_{x}^{\infty} a(u) \mathrm{d} u$ are subexponential, but for which $a(x) \notin 8^{*}$; see [6] for an example of such a distribution tail.

\subsection{A counterexample related to Proposition 2.4}

We use the same notation as in the previous subsection, but now assume that $\alpha=0$. Let $a(x)$ be a distribution tail which is long tailed, but not in 8 . In addition, assume that

$$
\limsup _{x \rightarrow \infty} \int_{0}^{x} \frac{a(x-y)}{a(x)} \mathrm{d} a(y)=\infty .
$$

(All known examples of distributions in $\mathscr{L} \backslash \&$ satisfy this property; see Subsection 3.1.) Since $a(x)$ is long tailed, there exists a function $h(x)$ such that $h(x) \rightarrow \infty, a(x-h(x)) \sim a(x)$, and

$$
\limsup _{x \rightarrow \infty} \int_{h(x)}^{x-h(x)} \frac{a(x-y)}{a(x)} \mathrm{d} a(y)=\infty .
$$

Now set $L(x)=a(\log x)$. From the previous considerations, it follows that there exists a function $d(x)$ such that we have $d(x) \rightarrow \infty, d(x)=\mathrm{o}(\sqrt{x}), L(x / d(x)) \sim L(x)$, and

$$
\limsup _{x \rightarrow \infty} \int_{d(x)}^{x / d(x)} \frac{L(x / y)}{L(x)} \mathrm{d} L(y)=: \limsup _{x \rightarrow \infty} r(x)=\infty .
$$

Let $x_{n}, n \geq 1$, be a sequence such that $x_{n} \rightarrow \infty$ and $r\left(x_{n}\right) \uparrow \infty$. 
We are now ready to define $X$ and $Y$. Let $X$ be a random variable with tail $L(x)$. Set, for $x \geq 0, s(x)=\sqrt{r\left(x_{n}\right)}$ when $x \in\left[d\left(x_{n}\right), d\left(x_{n+1}\right)\right)$. Let $\mathrm{P}\{Y>x\}=\mathrm{P}\{X>x\} / s(x)=$ $L(x) / s(x)$. Since $s(x) \uparrow \infty, \mathrm{P}\{Y>x\}=\mathrm{o}(\mathrm{P}\{X>x\})$. Conditioning on values of $X$, we obtain

$$
\begin{aligned}
\frac{\mathrm{P}\{X Y>x ; d(x)<X<x / d(x)\}}{\mathrm{P}\{X>x\}} & =\int_{d(x)}^{x / d(x)} \frac{L(x / y)}{s(x / y) L(x)} \mathrm{d} L(y) \\
& \geq \int_{d(x)}^{x / d(x)} \frac{L(x / y)}{s(d(x)) L(x)} \mathrm{d} L(y) \\
& =\frac{r(x)}{s(d(x))} .
\end{aligned}
$$

This is at least $\sqrt{r\left(x_{n}\right)}$ at the points $x_{n}, n \geq 1$. We conclude that

$$
\limsup _{x \rightarrow \infty} \frac{\mathrm{P}\{X Y>x ; X>d(x)\}}{\mathrm{P}\{X>x\}}=\infty,
$$

implying that (1.1) does not hold.

\subsection{A counterexample related to Proposition 2.3}

In this subsection we show that the condition $\mathrm{P}\{Y>x\}=\mathrm{o}(\mathrm{P}\{X>x\} / m(x))$ in Proposition 2.3 cannot be weakened to the more appealing condition $\mathrm{P}\{Y>x\}=\mathrm{o}(\mathrm{P}\{X>x\})$. To construct a counterexample, we let

$$
f_{X}(x)=\frac{1}{x^{2} \log x}
$$

be the density of $X$. In this case $\mathrm{P}\{X>x\} \sim 1 / x \log x$ and $\mathrm{P}\{U>x\} \sim 1 / x$. Furthermore, let

$$
f_{Y}(y)= \begin{cases}f_{Y}\left(\sqrt{x_{n+1}}\right), & x_{n} \leq y<\sqrt{x_{n+1}}, \\ \frac{1}{y^{2} \log y \log \log y}, & \sqrt{x_{n+1}} \leq y<x_{n+1},\end{cases}
$$

be the density of $Y$. Here, $x_{n}=\exp \left(\exp \left(n^{2}\right)\right)$. It is clear that

$$
\begin{aligned}
\mathrm{P}\{Y>x\} & =\int_{x}^{\infty} f_{Y}(y) \mathrm{d} y \\
& <\int_{x}^{\infty} \frac{1}{y^{2} \log y \log \log y} \mathrm{~d} y \\
& \sim \frac{1}{x \log x \log \log x} \\
& =\mathrm{o}(\mathrm{P}\{X>x\}) .
\end{aligned}
$$

Also,

$$
\begin{aligned}
\mathrm{E}\{Y\} & =\sum_{n=0}^{\infty}\left(\int_{x_{n}}^{\sqrt{x_{n+1}}}+\int_{\sqrt{x_{n+1}}}^{x_{n+1}}\right) y f_{Y}(y) \mathrm{d} y \\
& =\sum_{n=0}^{\infty} f_{Y}\left(\sqrt{x_{n+1}}\right) \frac{x_{n+1}-x_{n}^{2}}{2}+\sum_{n=0}^{\infty}\left(\log \log \log x_{n+1}-\log \log \log \sqrt{x_{n+1}}\right) .
\end{aligned}
$$


The first sum can be bounded by

$$
\sum f_{Y}\left(\sqrt{x_{n+1}}\right) x_{n+1}=\sum \frac{1}{\log \sqrt{x_{n+1}} \log \log \sqrt{x_{n+1}}}<\sum \frac{2}{\exp \left(n^{2}\right)}<\infty .
$$

To bound the second sum, note that

$$
\log \log \log x_{n+1}-\log \log \log \sqrt{x_{n+1}}=\log \frac{n^{2}}{n^{2}-\log 2} \sim \frac{\log 2}{n^{2}} .
$$

Thus, the second sum is finite as well, and we conclude that $\mathrm{E}\{Y\}<\infty$. Summing up all these facts, we have: $U$ is a regularly varying random variable, $\mathrm{P}\{Y>x\}=\mathrm{o}(\mathrm{P}\{X>x\})$, and $\mathrm{E}\{Y\}<\infty$. Now we will show that (1.1) does not hold.

It is sufficient to show that

$$
\lim _{M \rightarrow \infty} \liminf _{x \rightarrow \infty} \int_{M}^{g(x) x} \frac{\mathrm{P}\{U>\log x-\log y\}}{\mathrm{P}\{U>\log x\}} y \mathrm{dP}\{Y \leq y\}>0 .
$$

We have

$$
\begin{aligned}
\int_{M}^{g\left(x_{n}\right) x_{n}} & \frac{\mathrm{P}\left\{U>\log x_{n}-\log y\right\}}{\mathrm{P}\left\{U>\log x_{n}\right\}} y \mathrm{dP}\{Y \leq y\} \\
& >\int_{\sqrt{x_{n}}}^{g\left(x_{n}\right) x_{n}} \frac{\mathrm{P}\left\{U>\log x_{n}-\log y\right\}}{\mathrm{P}\left\{U>\log x_{n}\right\}} y \mathrm{dP}\{Y \leq y\} \\
& =\log x_{n} \int_{\sqrt{x_{n}}}^{g\left(x_{n}\right) x_{n}} \frac{1}{\log x_{n}-\log y} \frac{\mathrm{d} y}{y \log y \log \log y} \\
> & \log x_{n} \int_{\sqrt{x_{n}}}^{g\left(x_{n}\right) x_{n}} \frac{1}{\log x_{n}-\log y} \frac{\mathrm{d} y}{y \log x_{n} \log \log x_{n}} \\
= & \frac{1}{\log \log x_{n}} \int_{\sqrt{x_{n}}}^{g\left(x_{n}\right) x_{n}} \frac{1}{\log x_{n}-\log y} \frac{\mathrm{d} y}{y} \\
= & \frac{\log \log \sqrt{x_{n}}-\log \log \left(1 / g\left(x_{n}\right)\right)}{\log \log x_{n}} .
\end{aligned}
$$

Now note that we can choose a $g(x)$ which tends to 0 very slowly, e.g. $g(x)>1 / \log x$. In this case

$$
\frac{\log \log \sqrt{x_{n}}-\log \log \left(1 / g\left(x_{n}\right)\right)}{\log \log x_{n}} \rightarrow 1 .
$$

Therefore, (3.1) holds which implies that (1.1) does not hold.

\section{Application to a random difference equation}

Let $R$ be a random variable satisfying (1.2), let $0 \leq M \leq 1, \mathrm{P}\{M=1\}=0$, and let $Q$ be independent of $M$. Throughout this section, we assume that $\mathrm{P}\{Q>x\}=g(x) \mathrm{e}^{-\alpha x}$, with $\alpha>0$ and $g(\log x)$ slowly varying. Clearly this assumption implies that $\mathrm{e}^{Q}$ is regularly varying with index $-\alpha$. Our main interest is in obtaining the tail behavior of $R$. Before we proceed with our analysis, we mention some related work on this problem. Without dependence and nonnegativity assumptions on $Q$ and $M$, logarithmic asymptotics for $R$ have been obtained 
in [11]. Precise asymptotics in the present setting, with $Q$ exponentially distributed, have been obtained in [19].

The goal of this section is to relax the assumption on $Q$ made in [19], and to give an illustration of the applicability of the results obtained in previous sections: in the next four subsections we give applications of Propositions 2.1-2.4.

\subsection{An application of Proposition 2.1}

If the function $g(x)$ is bounded away from 0 , it is straightforward to obtain the tail behavior of $R$, as shown by the following result.

Proposition 4.1. Assume that $\lim \sup _{x \rightarrow \infty} \sup _{1 \leq y \leq x} g(y) / g(x)<\infty$. Then

$$
\mathrm{P}\{R>x\} \sim \mathrm{E}\left\{\mathrm{e}^{\alpha M R}\right\} \mathrm{P}\{Q>x\}
$$

if and only if $\mathrm{E}\left\{\mathrm{e}^{\alpha M Q}\right\}<\infty$.

Proof. The proof is similar to the proof of Theorem 5.1 of [19]. Along the lines of Proposition 5.1 of that paper, it can be shown that $\mathrm{E}\left\{\mathrm{e}^{\alpha M R}\right\}<\infty$ if and only if $\mathrm{E}\left\{\mathrm{e}^{\alpha M Q}\right\}<\infty$. Taking exponents on both sides in (1.2), we obtain $\mathrm{e}^{R} \stackrel{\mathrm{D}}{=} \mathrm{e}^{M R} \mathrm{e}^{Q}$. We see that all conditions of Proposition 2.1, with $L(x)=g(\log x)$, are satisfied, providing the result.

The above result covers the case where the limit of $g(x)$ exists and is strictly positive (possibly $\infty$ ). As we will see below, the case in which $g(x) \rightarrow 0$ is more challenging to deal with.

\subsection{An application of Proposition 2.2}

If $g(x) \rightarrow 0$, the main difficulty is to show that $M R$ is sufficiently light tailed. In the setting of Proposition 2.2 this is possible under the following reasonable assumptions.

Proposition 4.2. Suppose that $Q \in \delta(\alpha), \alpha>0$. Then $\mathrm{E}\left\{\mathrm{e}^{\alpha M R}\right\}<\infty$ and $\mathrm{P}\{R>x\} \sim$ $\mathrm{E}\left\{\mathrm{e}^{\alpha M R}\right\} \mathrm{P}\{Q>x\}$.

Proof. Since $\mathrm{E}\left\{\mathrm{e}^{\alpha Q}\right\}<\infty$ and $R \stackrel{\mathrm{D}}{=} M R+Q$, the statement follows from Proposition 2.2 after we have verified that

$$
\mathrm{P}\{M R>x\}=\mathrm{o}(\mathrm{P}\{Q>x\}) .
$$

For this, we first use a similar bounding procedure as in Proposition 5.1 of [19]. Let $M_{n}, n \geq 0$, and $Q_{n}, n \geq 0$, be mutually independent i.i.d. copies of $M$ and $Q$, respectively. Then we can write $M R \stackrel{\bar{D}}{=} \sum_{k=1}^{\infty} Q_{k} \prod_{i=1}^{k} M_{i}$. Define the sequence of random times $\bar{\tau}_{k}, k \geq 0$, as follows. Let $\bar{\tau}_{0}=0$ and, for $k \geq 1$,

$$
\bar{\tau}_{k}=\inf \left\{n>\bar{\tau}_{k-1}: M_{n} \leq \eta\right\} .
$$

Take $\eta>0$ small enough so that $\mathrm{P}\{M>\eta\} \mathrm{E}\left\{\mathrm{e}^{\alpha} Q\right\}<1$, and write

$$
M R \stackrel{\mathrm{D}}{=} \sum_{k=1}^{\infty} Q_{k} \prod_{i=1}^{k} M_{i}=\sum_{k=1}^{\infty} \sum_{n=\bar{\tau}_{k-1}+1}^{\bar{\tau}_{k}} Q_{n} \prod_{i=1}^{n} M_{i} \leq M_{1} \sum_{k=1}^{\infty} \eta^{k-1} \sum_{n=\bar{\tau}_{k-1}+1}^{\bar{\tau}_{k}} Q_{n} .
$$

Set $C_{k}=\sum_{n=\bar{\tau}_{k-1}+1}^{\bar{\tau}_{k}} Q_{n}$. The sequence $\left\{C_{k}, k \geq 1\right\}$ is i.i.d., and since $Q_{n} \in \&(\alpha)$, we have, using a well-known result on geometric random sums (see, e.g. [4]),

$$
\mathrm{P}\left\{C_{k}>x\right\} \sim c_{\eta} \mathrm{P}\{Q>x\},
$$


with $c_{\eta}$ a finite constant. Set $R_{\eta}=\sum_{k=1}^{\infty} \eta^{k-1} C_{k}$. Observe that $R$ is stochastically dominated by $R_{\eta}$ and that $R_{\eta} \stackrel{\mathrm{D}}{=} \eta R_{\eta}+C_{1}$. This is an equation similar to the original equation (1.2), but with $Q$ replaced by $C_{1}$ and $M$ replaced by $\eta$. We see that

$$
\mathrm{E}\left\{\exp \left(s R_{\eta}\right)\right\}=\prod_{n=1}^{\infty} \mathrm{E}\left\{\exp \left(s \eta^{n-1} C_{n}\right)\right\}
$$

from which it simply follows that there exists a $\delta>0$ such that $\operatorname{E}\left\{\exp \left((\alpha+\delta) \eta R_{\eta}\right)\right\}<\infty$.

Consequently, by the classical version of Breiman's theorem we obtain

$$
\mathrm{P}\left\{R_{\eta}>x\right\} \sim \mathrm{E}\left\{\exp \left(\alpha \eta R_{\eta}\right)\right\} \mathrm{P}\left\{C_{1}>x\right\} \sim \mathrm{E}\left\{\exp \left(\alpha \eta R_{\eta}\right)\right\} c_{\eta} \mathrm{P}\{Q>x\} .
$$

Since $M<1$ a.s. and since $\mathrm{P}\{Q>x / \zeta\}=\mathrm{o}(\mathrm{P}\{Q>x\})$, we conclude, from the tail asymptotics for $R_{\eta}$, that, for any $\zeta<1$,

$$
\begin{aligned}
\mathrm{P}\left\{M R_{\eta}>x\right\} & \leq \mathrm{P}\{M>\zeta\} \mathrm{P}\left\{R_{\eta}>x\right\}+\mathrm{P}\left\{R_{\eta}>\frac{x}{\zeta}\right\} \\
& =\mathrm{o}(\mathrm{P}\{Q>x\}) \quad \text { as } \zeta \uparrow 1 .
\end{aligned}
$$

Since $R$ is stochastically dominated by $R_{\eta}$, we arrive at (4.1).

\subsection{An application of Proposition 2.3}

To check the sufficient condition of Proposition 2.3 requires more work. We therefore focus on a special case. To save space, we leave out some of the details which are straightforward or which overlap with similar arguments given before.

Proposition 4.3. Assume that $\mathrm{P}\{1-M \leq x\}=h(1 / x) x^{\gamma}$ for some $\gamma>0$ and a function $h$ which is slowly varying at $\infty$. Assume, in addition, that $\mathrm{P}\{Q>x\} \sim \ell(x) x^{-\beta} \mathrm{e}^{-\alpha x}$, with $\ell$ a slowly varying function and $\beta \in(0,1)$. Then $\mathrm{E}\left\{\mathrm{e}^{\alpha M R}\right\}<\infty$ and $\mathrm{P}\{R>x\} \sim$ $\mathrm{E}\left\{\mathrm{e}^{\alpha M R}\right\} \mathrm{P}\{Q>x\}$ if $\beta+\gamma>1$.

The proof of this proposition relies on the following lemma.

Lemma 4.1. Under the assumptions of Proposition 4.3, for any $\eta \in[0,1)$,

$$
\mathrm{P}\{M Q>x \mid M>\eta\} \sim \frac{1}{\mathrm{P}\{M>\eta\}} \Gamma(1+\gamma)(\alpha)^{-\gamma} h(x) \ell(x) x^{-\gamma-\beta} \mathrm{e}^{-\alpha x} .
$$

Proof. Note that the tail $1 /(1-M)$ is regularly varying at $\infty$ with index $-\gamma$. Therefore,

$$
\mathrm{P}\left\{\frac{1}{1-M}>x\right\} \sim \mathrm{P}\left\{\frac{1}{1-M}>x+1\right\}=\mathrm{P}\left\{\frac{M}{1-M}>x\right\} \text { for } x \rightarrow \infty .
$$

Consequently, if we define $Y=(1-M) / M$ then $\mathrm{P}\{Y \leq x\} \sim \mathrm{P}\{1-M \leq x\}=h(1 / x) x^{\gamma}$ as $x \downarrow 0$. Let $w(s)$ be the Laplace-Stieltjes transform of $Y$. By Fellers Tauberian theorem (see [1, Theorem 1.7.1]) we obtain, as $s \rightarrow \infty$,

$$
w(s) \sim \Gamma(1+\gamma) h(s) s^{-\gamma} .
$$

We see that $M=1 /(Y+1)$, so that

$$
\begin{aligned}
\mathrm{P}\{M Q>x\} & =\mathrm{P}\{Q>x(Y+1)\} \\
& =\mathrm{e}^{-\alpha x} x^{-\beta} \ell(x) \int_{0}^{\infty} \frac{\ell(x(y+1))}{\ell(x)}(y+1)^{-\beta} \mathrm{e}^{-\alpha x y} \mathrm{dP}\{Y \leq y\} .
\end{aligned}
$$


We say that $X \stackrel{\mathrm{D}}{\leq} Y$ if $\mathrm{P}(X>t) \leq \mathrm{P}(Y>t)$ for all $t \in \mathbb{R}$. Now, note that, for some $\varepsilon>0$,

$$
\begin{aligned}
\int_{0}^{\infty} & \frac{\ell(x(y+1))}{\ell(x)}(y+1)^{-\beta} \mathrm{e}^{-\alpha x y} \mathrm{dP}\{Y \leq y\} \\
& \sim \int_{0}^{1} \frac{\ell(x(y+1))}{\ell(x)}(y+1)^{-\beta} \mathrm{e}^{-\alpha x y} \mathrm{dP}\{Y \leq y\}+\mathrm{o}\left(\mathrm{e}^{-\varepsilon x}\right) \\
& \sim \int_{0}^{1}(y+1)^{-\beta} \mathrm{e}^{-\alpha x y} \mathrm{dP}\{Y \leq y\}+\mathrm{o}\left(\mathrm{e}^{-\varepsilon x}\right) \\
& \sim \int_{0}^{\infty}(y+1)^{-\beta} \mathrm{e}^{-\alpha x y} \mathrm{dP}\{Y \leq y\}+\mathrm{o}\left(\mathrm{e}^{-\varepsilon x}\right) .
\end{aligned}
$$

In the first step we used the fact that the contribution of the integral from the interval $[1, \infty)$ is exponentially small, and in the second step we applied the uniform convergence theorem for slowly varying functions. From (4.3) we obtain, as $x \rightarrow \infty$,

$$
\begin{aligned}
\Gamma(1+\gamma) h(x)(\alpha x)^{-\gamma} & \sim w(\alpha x) \\
& =\int_{0}^{\infty} \mathrm{e}^{-\alpha x y} \mathrm{dP}\{Y \leq y\} \\
& \sim \int_{0}^{\infty}(y+1)^{-\beta} \mathrm{e}^{-\alpha x y} \mathrm{dP}\{Y \leq y\}
\end{aligned}
$$

The last equivalence can be obtained by noting that the main contribution to the asymptotics of the integral comes from $y \in[0, \delta]$, with arbitrarily small $\delta$, implying that $(y+1)^{-\beta}$ can be made arbitrary close to 1 for $y \in[0, \delta]$. Combining these results, we obtain the statement of the lemma for $\eta=0$. The extension to general $\eta$ is straightforward.

Proof of Proposition 4.3. Let $M^{\eta}$ be a random variable distributed as $M \mid M>\eta$. Since $c \ell(x) h(x) x^{-\gamma-\beta} \in \S_{d}$ for any constant $c>0$, we find, by Theorem 2.1 of [16] and Lemma 4.1, that $M^{\eta} Q \in \delta(\alpha)$ for any $\eta<1$. Now we proceed similarly, but slightly differently as in the proof of Proposition 4.2; the notation introduced in that proof is used here as well. We can bound $M R$ in a similar but slightly more precise way, as in (4.2), to obtain

$$
M R \stackrel{\mathrm{D}}{=} \sum_{k=1}^{\infty} Q_{k} \prod_{i=1}^{k} M_{i}=\sum_{k=1}^{\infty} \sum_{n=\bar{\tau}_{k-1}+1}^{\bar{\tau}_{k}} Q_{n} \prod_{i=1}^{n} M_{i} \leq \sum_{k=1}^{\infty} \eta^{k-1} \sum_{n=\bar{\tau}_{k-1}+1}^{\bar{\tau}_{k}} M_{n} Q_{n}
$$

Define $\bar{C}_{k}=\sum_{n=\bar{\tau}_{k-1}+1}^{\bar{\tau}_{k}} M_{n} Q_{n}$. It is clear that $\bar{C}_{k}, k \geq 1$, is an i.i.d. sequence. Let $M_{i}^{\eta}, i \geq 1$, be an i.i.d. sequence independent of everything else such that $M_{1}^{\eta} \stackrel{D}{=} M_{1} \mid M_{1}>\eta$. Then, we see that

$$
\bar{C}_{1} \stackrel{\mathrm{D}}{\leq} \sum_{n=1}^{\bar{\tau}_{1}} M_{n}^{\eta} Q_{n}
$$

Since $M_{n}^{\eta} Q_{n} \in \delta(\alpha)$, we can proceed as in the proof of Proposition 4.2. Since $\bar{\tau}_{1}$ is independent of the sequence $\left(M_{n}^{\eta}\right), \bar{C}_{1}$ has the same tail behavior as $M_{1}^{\eta} Q_{1}$. As before, $R$ is stochastically smaller than $R_{\eta}=\sum_{n=1}^{\infty} \eta^{n-1} \bar{C}_{n}$, and as before we can show that $R_{\eta}$ has the same tail behavior as $\bar{C}_{1}$. We conclude that $\mathrm{P}\{M R>x\}=\mathrm{O}(\mathrm{P}\{M Q>x\})$. This enables us to apply 
Proposition 2.3. Define $X=\mathrm{e}^{Q}$. Then, integrating by parts,

$$
\begin{aligned}
m(x) & =\int_{0}^{x} t^{\alpha-1} \mathrm{P}\{X>t\} \mathrm{d} t-x^{\alpha} \mathrm{P}\{X>x\} \\
& =\int_{0}^{x} t^{-1} \ell(\log t)(\log t)^{-\beta} \mathrm{d} t-(1+\mathrm{o}(1))(\log x)^{-\beta} \ell(\log x) \\
& \sim \int_{\log t=0}^{\log x} \ell(\log t)(\log t)^{-\beta} \mathrm{d} \log t-(1+\mathrm{o}(1))(\log x)^{-\beta} \ell(\log x) \\
& \sim \frac{1}{1-\beta}(\log x)^{1-\beta} \ell(\log x),
\end{aligned}
$$

where we applied Karamata's theorem in the last step. Thus, $m(x) / \mathrm{P}\{X>x\} \sim$ $(1 /(1-\beta)) x^{\alpha} \log x$. Writing $Y=\mathrm{e}^{M R}$, we see that, as $x \rightarrow \infty$,

$$
\begin{aligned}
\mathrm{P}\{Y>x\} & =O\left(\mathrm{P}\left\{\mathrm{e}^{M Q}>x\right\}\right) \\
& =\mathrm{O}\left(x^{-\alpha}(\log x)^{-\gamma-\beta} \ell(\log x) h(\log x)\right),
\end{aligned}
$$

which implies condition (2.2), since $\gamma+\beta>1$. We thus conclude, from Proposition 2.3, that (1.1) holds for our choice of $X$ and $Y$, which implies our assertion.

\subsection{An application of Proposition 2.4}

If $\alpha=0, Q$ is heavy tailed. Results for regularly varying $Q$ can be found in [13], [17], and [20]. Here we focus on the case in which the tail of $Q$ is lighter than any power tail.

Proposition 4.4. If $Q \in S$ and in the domain of attraction of the Gumbel law then

$$
\mathrm{P}\{R>x\} \sim \mathrm{P}\{Q>x\} .
$$

This result is fundamentally different from the case in which $Q$ is regularly varying with index $-\alpha$, in which case it is known, from the above references, that $\mathrm{P}\{R>x\} \sim$ $\left(1 /\left(1-\mathrm{E}\left\{M^{\alpha}\right\}\right)\right) \mathrm{P}\{Q>x\}$.

Proof of Proposition 4.4.. Using the same arguments as in Proposition 4.2 (note that $Q \in$ $8(0)$ ), we obtain $\mathrm{P}\{R>x\} \leq \mathrm{P}\left\{R_{\eta}>x\right\}$, with $R_{\eta}=\sum_{n=1}^{\infty} \eta^{n-1} C_{n}$, with $\mathrm{P}\left\{C_{1}>x\right\} \sim$ $c(\eta) \mathrm{P}\{Q>x\}$. This implies that $C_{1}$ is subexponential and in the domain of attraction of the Gumbel distribution as well. This allows us to apply Lemma A3.27 of [8] to obtain $\mathrm{P}\left\{R_{\eta}>x\right\} \sim$ $\mathrm{P}\left\{C_{1}>x\right\}$. Thus, $\mathrm{P}\{M R>x\}$ is asymptotically smaller than $c(\eta) \mathrm{P}\{M Q>x\}$. Since $Q$ is in the domain of attraction of the Gumbel law, the tail of $Q$ is also of rapid variation, implying that $\mathrm{P}\{M Q>x\}=\mathrm{o}(\mathrm{P}\{Q>x\})$. Hence, $\mathrm{P}\{M R>x\}=\mathrm{o}(\mathrm{P}\{Q>x\})$ also. The proof is now completed by applying Proposition 2.4 .

\section{Concluding remarks}

In this paper we derived several extensions of Breiman's theorem, by introducing specific assumptions on the slowly varying function $L$. An interesting question which we did not resolve is whether the condition that the slowly varying function $L$ is also of $\Pi$-variation (see [1, Chapter 3]) would be sufficient for (1.1) to hold. That the assumptions on $L$ are in some sense necessary was illustrated in Section 3. In Section 4 we applied our results from Section 2 to analyze the equation $R \stackrel{\mathrm{D}}{=} M R+Q$. We assumed that $Q$ and $M$ were independent, which may 
be too restrictive in some applications. It would therefore be interesting to extend Breiman's theorem to the case where $X$ and $Y$ are dependent. A partial result in this direction has recently been obtained in [18].

\section{Acknowledgements}

The research of Denis Denisov was supported by the Dutch BSIK project (BRICKS) and the EURO-NGI project. Both authors are indebted to an anonymous referee for uncovering an error in a previous version of the manuscript and to EURANDOM, where this research was carried out.

\section{References}

[1] Bingham, N. H., Goldie, C. M. And Teugels, J. L. (1987). Regular Variation. Cambridge University Press.

[2] Breiman, L. (1965). On some limit theorems similar to the arc-sin law. Theory Prob. Appl. 10, 323-331.

[3] Cline, D. B. H. (1986). Convolution tails, product tails and domains of attraction. Prob. Theory Relat. Fields 72, 529-557.

[4] Cline, D. B. H. (1987). Convolutions of distributions with exponential and subexponential tails. J. Austral. Math. Soc. Ser. A 43, 347-365.

[5] Cline, D. B. H. and Samorodnitsky, G. (1994). Subexponentiality of the product of independent random variables. Stoch. Process. Appl. 49, 75-98.

[6] Denisov, D., Foss, S. And Korshunov, D. (2004). Tail asymptotics for the supremum of a random walk when the mean is not finite. Queueing Systems 46, 15-33.

[7] Embrechts, P. And Goldie, C. M. (1980). On closure and factorization properties of subexponential and related distributions. J. Austral. Math. Soc. Ser. A 29, 243-256.

[8] Embrechts, P., Klüppelberg, C. and Mikosch, T. (1997). Modelling Extremal Events. Springer, New York.

[9] Foss, S. And Korshunov, D. (2007). Lower limits and equivalences for convolution tails. Ann. Prob. 35, $366-383$.

[10] Goldie, C. M. (1991). Implicit renewal theory and tails of solutions of random equations. Ann. Appl. Prob. 1, $126-166$.

[11] Goldie, C. M. and Grübel, R. (1996). Perpetuities with thin tails. Adv. Appl. Prob. 28, 463-480.

[12] Gomes, M. I., de HaAn, L. And Pestana, D. (2004). Joint exceedances of the ARCH process. J. Appl. Prob. 41, 919-926. (Correction: 43 (2006), 1206.)

[13] Grey, D. R. (1994). Regular variation in the tail behaviour of solutions of random difference equations. Ann. Appl. Prob. 4, 169-183.

[14] Kesten, H. (1973). Random difference equations and renewal theory for products of random matrices. Acta Math. 131, 207-248.

[15] Klüppelberg, C. (1988). Subexponential distributions and integrated tails. J. Appl. Prob. 25, $132-141$.

[16] Klüppelberg, C. (1989). Subexponential distributions and characterizations of related classes. Prob. Theory Relat. Fields 82, 259-269.

[17] Konstantinides, D. G. and Miкosch, T. (2005). Large deviations and ruin probabilities for solutions to stochastic recurrence equations with heavy-tailed innovations. Ann. Prob. 33, 1992-2035.

[18] Maulik, K. And Resnick, S. (2005). Characterizations and examples of hidden regular variation. Extremes 7, 31-67.

[19] Maulik, K. and Zwart, B. (2006). Tail asymptotics of exponential functionals of Lévy processes. Stoch. Process. Appl. 116, 156-177.

[20] Resnick, S. I. AND Willekens, E. (1991). Moving averages with random coefficients and random coefficient autoregressive models. Commun. Statist. Stoch. Models 7, 511-525. 